SIAC-PUB-107

May 1965

EFFICIFINCY OF SECONDARY EIECTRON EMISSION MONITORS FOR $70 \mathrm{MeV}$ ELECTRUNS

\author{
S. A. Blankenburg, J.K. Cobb and J.J. Muray ${ }^{\dagger}$ \\ Stanford Linear Accelerator Center \\ Stanford University, Stanford, California
}

\title{
SUMM RY
}

The secondary electron emission coefficient from several elements in the form of thin foils has been measured using $70 \mathrm{MeV}$ electrons as bombarding particles.

Using foils with different atomic numbers, it was found that the secondary cmission coefficient per target elecuron in lie metal is noticeably larger for light elements, especially in cases of beryllium and aluminum. This indicates that metal oxide on the foil surface (Malter effect) is playing a dominant role in the secondary emission of these metals.

For other metals, the experimental results seem to indicate a relatively :small variation in the secondary emission coefficient per target electron, Iess than that predicted by V. J. Vanhuyse and R. E. Van de Vijver, but with the same general behavior.

The lack of thickness dependence in the case of tantalum foils is in agreement with the extensive experimental work of $B$. Planskoy and with the theoretical treatment of the secondary emission by Aggson.

The secondary electron emission coefficients will be given for the measured foils and the experimental values will be compared with the existing theories.

(This paper to be submitted Nuclear Instruments and Methods.)

\footnotetext{
* Work supported by U. S. Atomic Energy Commission.

tNow at Hewlett-Packard Company, Palo Alto, California.
} 


\section{INTRODUCTION}

The purpose of this paper is to summarize the experimental results on the secondary electron emission yields from thin metal foils bombarded with a bigh energy electron beam. This study was startfa as a search for a stable and accurate beam current monitor for high energy ( $10 \mathrm{MeV}-20 \mathrm{GeV}$ ) and high intensity $\left(10^{-11}-10^{-4}\right.$ amps $)$ electron beams.

\section{EXPERTMENTAL RESULTS}

\section{Experimental Setup}

For the experimental work to study the secondary eleatron emission properties of thin metal foils bombarded with electrons of $70 \mathrm{MeV}$ of energy, the Stanford Mark IV Iinear accelerator was used. The configuration of the apparatus used in the experiment is shown schematically in Fig. 1 . The electron beam from the accelerator was energy analyzed (energy resolution $\approx 1 \%$ ) by a magnetic deflection system, passed through two SEM's built from different foils, and finally collected in a Faraday cup. The efficiency ( $Y$ ) of the different foils is given as the ratio of charge integrated on a condenser to the charge collected in the Faraday cup. The Faraday cup was designed to catch more than $99 \%$ of the electrons in the beam.

The experiments were run under high vacuum sonditions, usually $3 \times 10^{-7}$ torr or better. The foils in the monitor were cleaned and the whole monitor, built from stainless steel, was baked out under vacuum for at least 12 hours. ${ }^{1}$ Yield Dependence on Atomic Number

Twelve different foils have been measured in this secondary emission study: Beryllium, aluminum, titanium, 320 stainless steel, nickel, copper, 
molybdenum, rhodium, silver, tantalum, wolfram, and gold. The results are displayed in Fig. 2 which shows the efficiency of each element as compared to the efficiency of gold. The upper curve shows the theoretical prediction of $\mathrm{V}$. J. Vanhuyse and R. E. Van de Vijver. ${ }^{2}$ A more detailed comparison of the experimental results with the theoretical predictions will be treated later. The interesting aspects of fecculin emizaion are more clearly seen in Fig. 3. Here the measured relative yields (Efficiency X/Efficiency Au) are divided by the electron density $\delta N_{0} Z / A$ in the foils, where $N_{0}$ is Avogadro's number, $\delta$ is the density of the foll, $z$ is the atomic number, and $A$ is the atomic weight. This curvo shows the efficiency of secondary electron emission per target electron. The light eleinents, especially beryllium and aluminum, are noticeably more efficient elements than theory predicts. Beginning with titanium and for $z$ higher than titanium, the efficiency is in good agreement with theory. Because both beryllium and aluminum have oxide coatings under normal conditions, this may indicate that the oxide is :playing a dominant role in the secondary emission of these metals. Except for these two metal foils, however, the experimental results seem to indicate a relatively small variation in efficiency per electron among elements, less than that predicted by theory but with the same general behavior.

\section{Thickness Dependence}

The secondary emission yield was measured for tantalum foils 1.0 mil and 2.4 mils thick. No significant difference was observed in their secondary emission for collection voltages between $I$ and 500 volts. This is a direct contradiction to the theory of Vanhuyse and Van de Vijver, which predicts that the total yield of a given foil goes as $Y=F_{1}$ (energy, metal constants) $+F_{2}$ (energy, metal constants) (thickness) $)^{\frac{1}{2}}$ where $F_{2}$ is larger than $F_{1}$ 
for all measured elements. For tantalum, this theory predicts that

$$
\frac{\mathrm{Y}_{\mathrm{Ta}}(2.4 \mathrm{miI})}{\mathrm{Y}_{\mathrm{Ta}}(1.0 \mathrm{mil})}=1.48
$$

This lack of thickness dependence is in agreement with the extensive experimental works of $B$. PLanskoy ${ }^{3}$ on aluminun and int the theoretical treatment of secondary electron emission by 'in. L. Aggson. ${ }^{4}$

Surface Effects

To investigate the effect of the beam on the surface layer of the foil, the yield was measured with different beam currents and varying collector voltage. In these runs the foil surface was "baked" out for an hour by beams of different currents values and the yield was recorded as a function of the collection voltage. Figure 4 shows the relative efficiency as a function of the collection voltage after an hour of baking with different intensity beams. It is evident from this figure that the yield changed at low collection voltage values; i.e., the energy spectrum of the secondary electrons is altered by the change in the surface layer, but the high voltage yield values did not change appreciably. This indicates that the low energy part of the secondary electron emission spectrum is a very sensitive function of the characteristics of the surface layer, but it does not influence the efficiency of the SEM when the collection voltage is high enough.

Figures 5 and 6 give the variation in efficiency as a function of collection voltage divided by the corresponding efficiency of gold and aluminum at the same voltage. These results were all obtained at energies around $70 \mathrm{MeV}$ with average currents between 3 and $5 \mu \mathrm{A}$. By taking the derivatives of these curves one can determine the energy spectrum of the secondary electrons from different metal foils as compared to gold and aluminum. Most of 
the electrons seem to be emitted with energies below $30 \mathrm{eV}$, which is what one should expect if the secondary emission is truly a surface phenomena, as seems to follow from the thickness dependance measurements.

There was no observed variation in the efficiency of secondary electron emission with currerts raneirs Irom $0.1 \mu \mathrm{A}$ to $20 \mu \mathrm{A}$. It was difficult to get consistent data be Low $0.1 \mu \mathrm{A}$ becsuse of the long integrating times involved and the galvanic currents of this magnitude observed on the Faraday cup itself from the cooling water. One of the most interesting aspects of the secondary emission is the small decrease in efficiency of the collection process from a maximum with increasing collection voltage. This effect has been explained by Aggson as a manifestation of the Malter effect due to the surface contamination by vacuum pump oils and the oxide coatings. In this study all the metal foils showed this effect to some degree, although the cleanest metal surfaces, particularly molybdenum, gold and tantalum, show exactly the same effect when compared to each other in the same run. For example, in Fig. 5 the efficiency of molybdenum foil divided by the efficiency of the gold foil in the same SEM during the same run remains constant above a 20-volt collection voltage. This would appear to indicate either that the effect is due to the experimental setup, or that both metals were contaminated exactly the same, possibly by vacuum pump oil.

\section{High Energy Secondary Electrons}

The properties of the high energy secondary electrons (knock-on electrons or delta rays) emitted from metal foils have been investigated by Shatas, Marshall and Pomerant ${ }^{5}$ and recently by $B$. Planskoy. ${ }^{3}$ It was found that the percentage of the high energy secondary electrons in the total yield depends on the thickness as $t^{\frac{1}{2}}$, and it is not influenced by the surface condition 
of the emitting foil. In this experiment the fraction of the high energy electrons emitted from the foil was estimated from the following measurements. On a threc-element SEM the collector voltages were applied with three different polarities, as shown in Figs. $7 a, 7 b$, and $7 \mathrm{c}$. Figure $7 \mathrm{a}$ shows the normal operation of a three-element SEM; in this case the electron collection efficiency is the largest and the messured yield dividud by two gives the efficiency per active foil surface. $I_{f}$ is the high energy component of the secondary emission current emitted by foil and unaffected by the field.

A fraction $\alpha$ of this high energy secondary electron current is stopped by the following foil. The arrows on Fig. 7 show the directions of the electron currents and the letters $F$ and $B$ refer to the front or back side emission currents relative to the beam direction.

Figure To shows the secondary electron currents when the first foil was negative with respect to the center and the third was at positive potential. Without high energy components in the emission, one would expect zero efficiency :with this polarity similar to the case where the first foil was positive and the third was negative with respect to the center foil (Fig. 7c). The experimental results from these measurements for $\mathrm{Zr}$ and Rd are shown in Fig. 8, where the electron collection efficiency on the center foil is plotted versus the collection voltage. It can be seen from these curves that the value of the collection voltage does not markedly change the collection efficiency in tile low voltage region as it does in the case of the low energy component. Using the measured electron collection efficiency, the fraction of the high energy component in the total electron collection efficiency can be estimated as

$$
\begin{gathered}
R_{\text {coll }}=\frac{\frac{1}{2}\left(\frac{Y_{R d+-}+Y_{R d-t}}{2}\right)}{Y_{R d}} \approx 3.6 \% \\
-6-
\end{gathered}
$$


This high energy electron collection efficiency might be interpreted as the lower limit for the ratio of the high to low energy electrons in the total secondary electron emission. 
I. S. A. Blankenburg, J.K. Cobb and J.J. Muray, IFEF Particle Accelerator Conference, Washington, D.C., March 10-12 (1965).

J.K. Cobb, J. J. Muray SLAC Report (to be published.)

2. V. J. Vanhuyse and F.E. Var d? Vijves, The. Tnstr. and Meth. 15, 63 (1962).

3. B. Planskoy, Nuc. Instr. and Meth. 24, 172 (1963).

4. Th. I. Aggson, Report IAL-1028, Laboratoire de I'accèlerateur linèaire, Orsay, France (1962).

5. R. A. Shatas, J. F. Marshall and M. A. Fumerantz, Phys. Rev. 96, 1199 (1954). 
1. Schematic of experimental setup.

2. Relative efficiency vs atomic number.

3. Relative efficiency per target eieutrus ri ctomic number.

4. $\frac{\mathrm{Y}_{\mathrm{Be}}}{\mathrm{Y}_{\mathrm{Al}}}$ vs collection voltage at $70 \mathrm{MeV}$.

5. Relative efficiency vs collection voltage. Primary electron energy $70 \mathrm{MeV}$.

6. Relative efficiency vs collection voltage.

7. Arrangements for measuring the hjgh energy components in the secondary electron emission.

8. Electron collection efficiency vs collection voltage. 


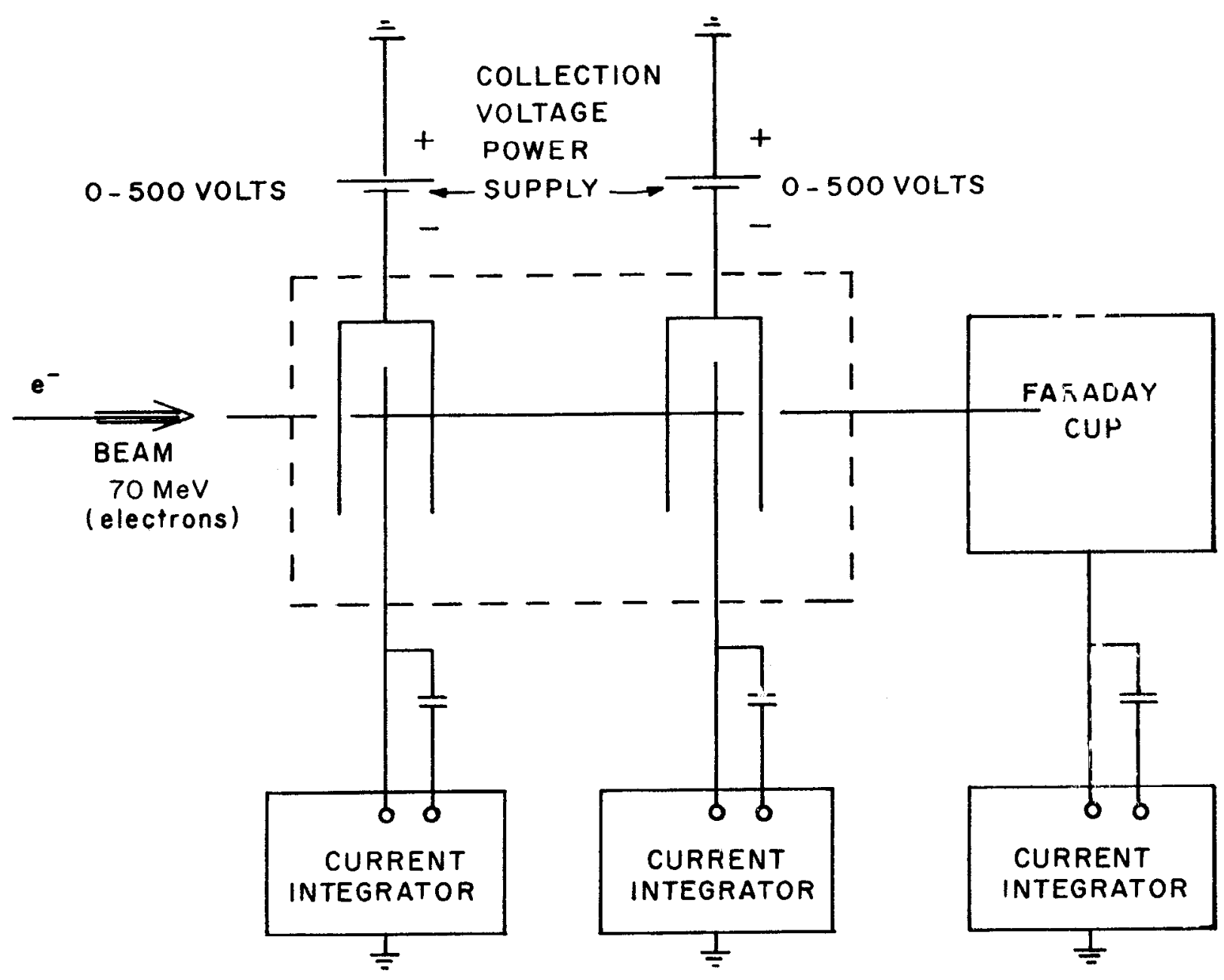

FIG. I - SCHEMATIC OF EXPERIMENTAL SETUP 


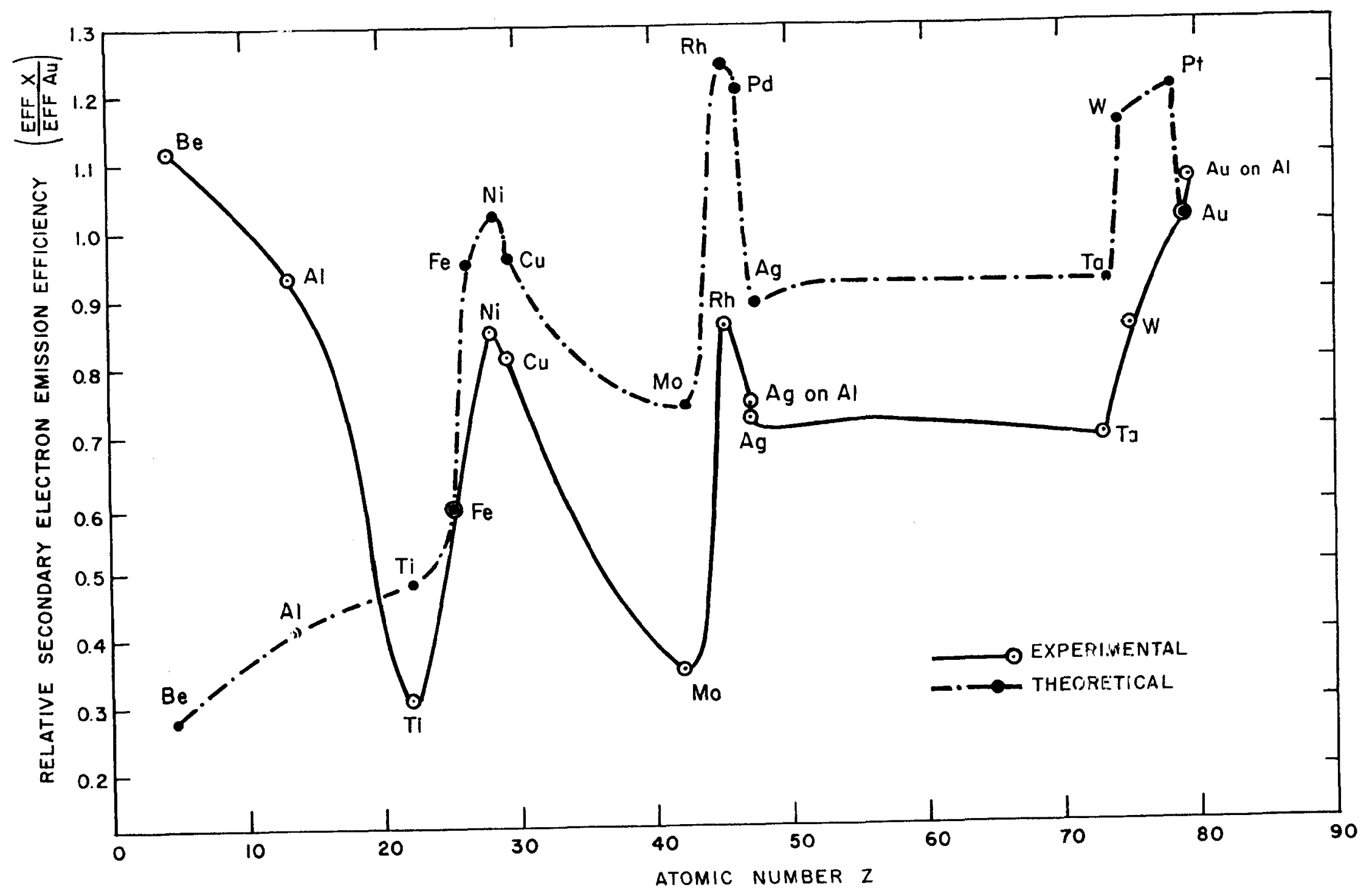

FIG. 2 - RELATIVE EFFICIENCY VS. ATOMIC NUMBER 


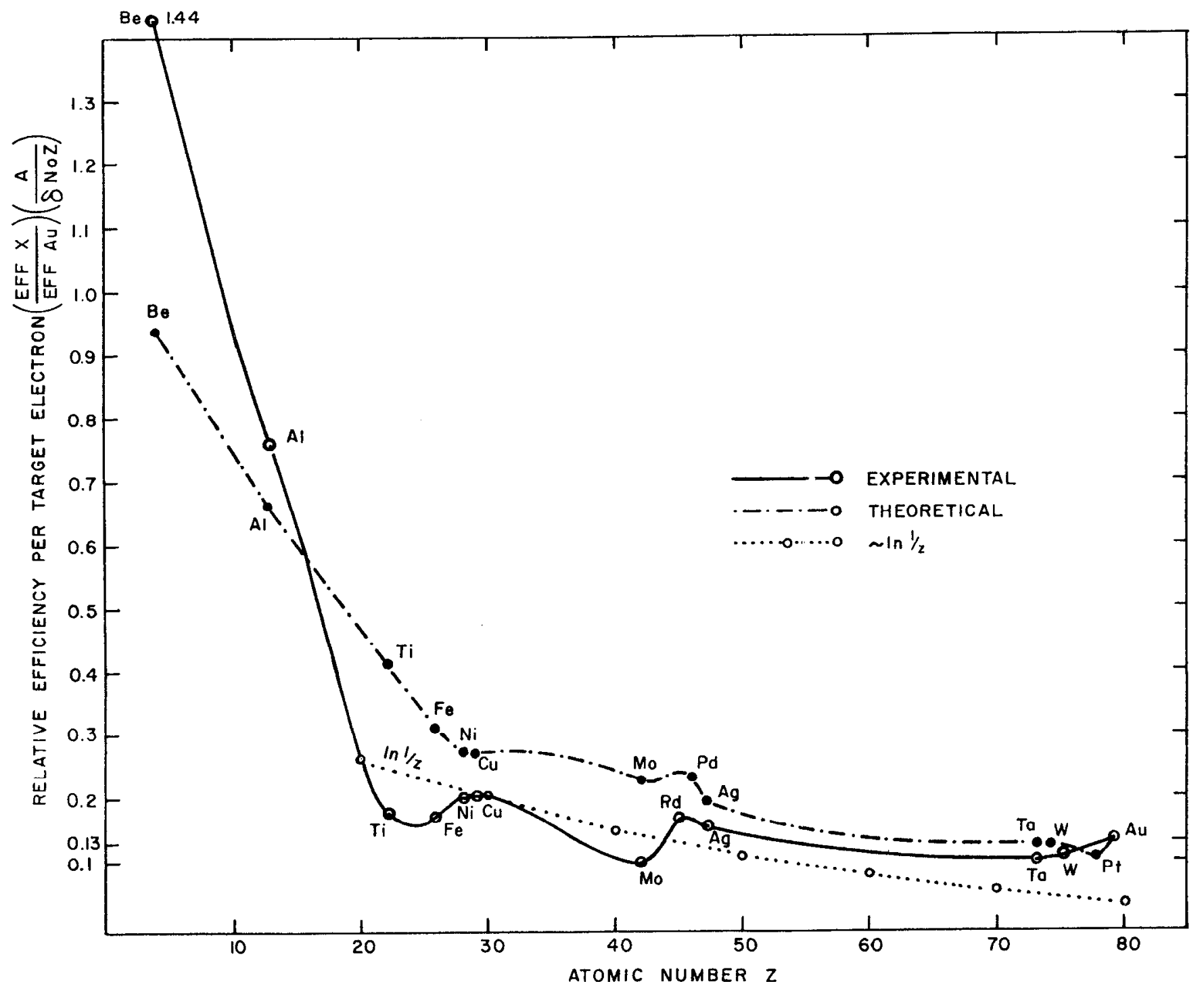

FIG. 3 - RELATIVE EFFICIENCY PER TARGET ELECTRON VS. ATOMIC NUMBER 


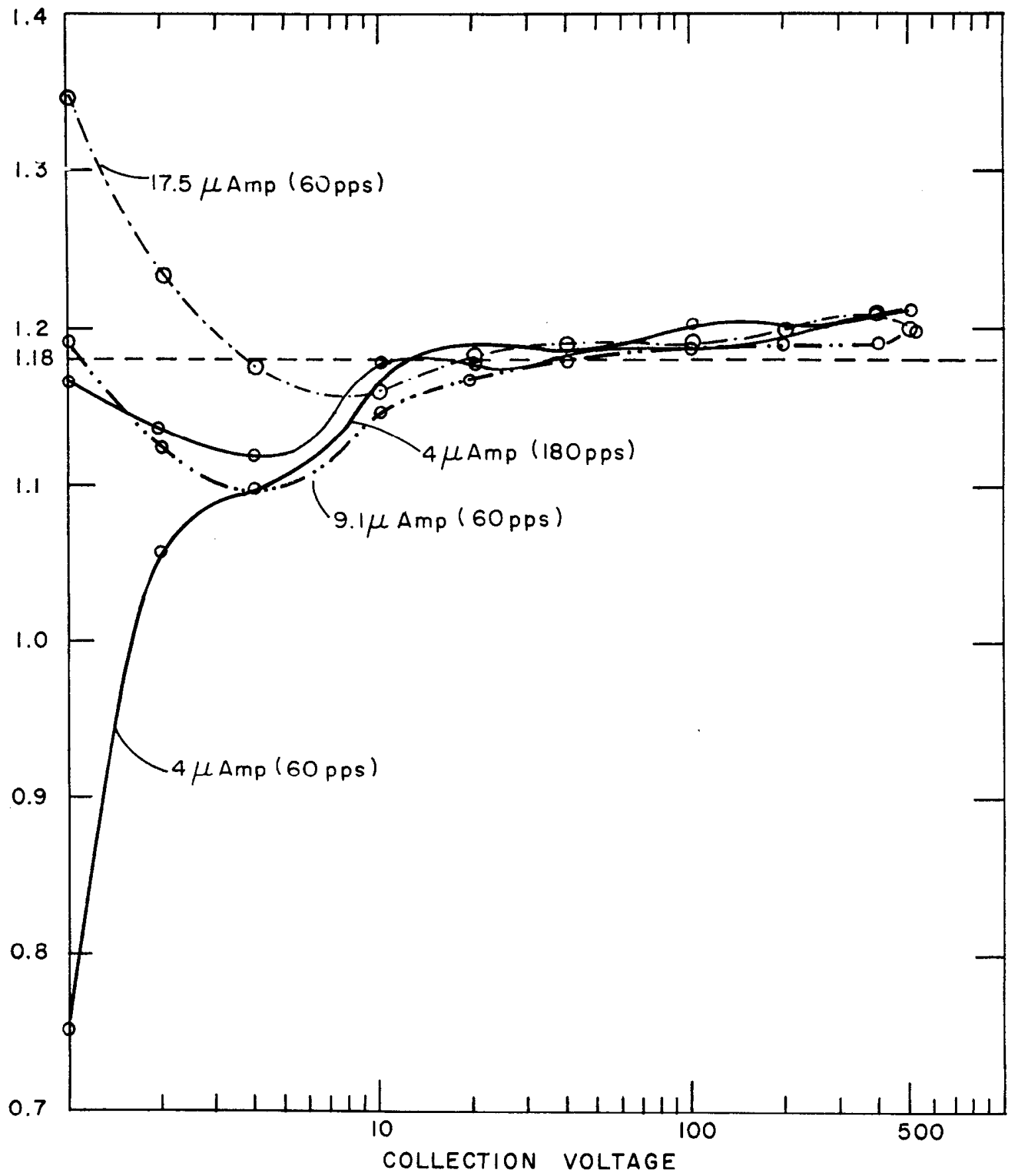

FIG. 4 - $\frac{Y_{8 E}}{Y_{A I}}$ VS. COLLECTION VOLTAGE AT $70 \mathrm{MeV}$. 


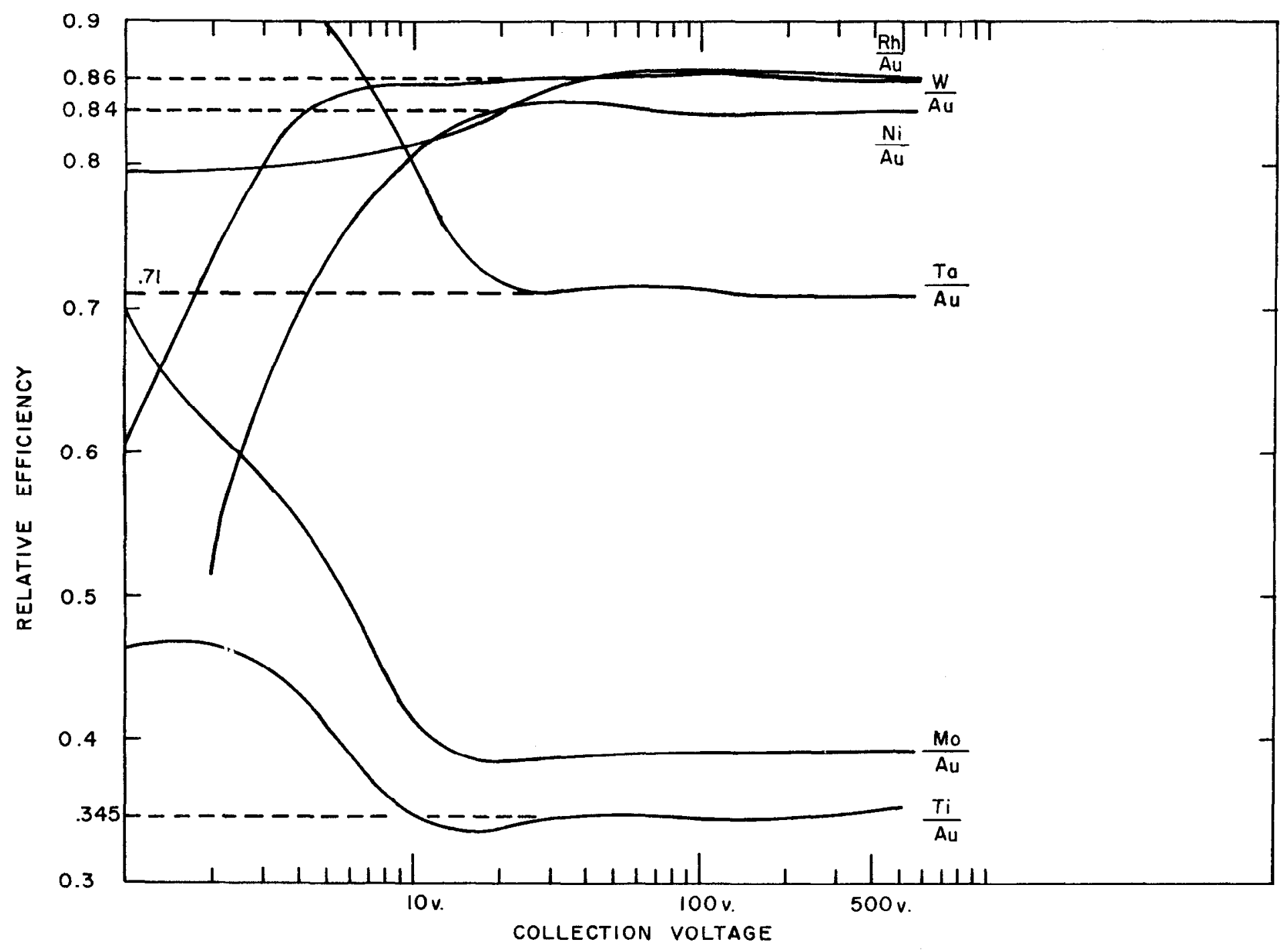

FIG. 5 - RELATIVE EFFICIENCY vS COLLECTION VOLTAGE. PRIMARY ELECTRON ENERGY $70 \mathrm{MeV}$. 


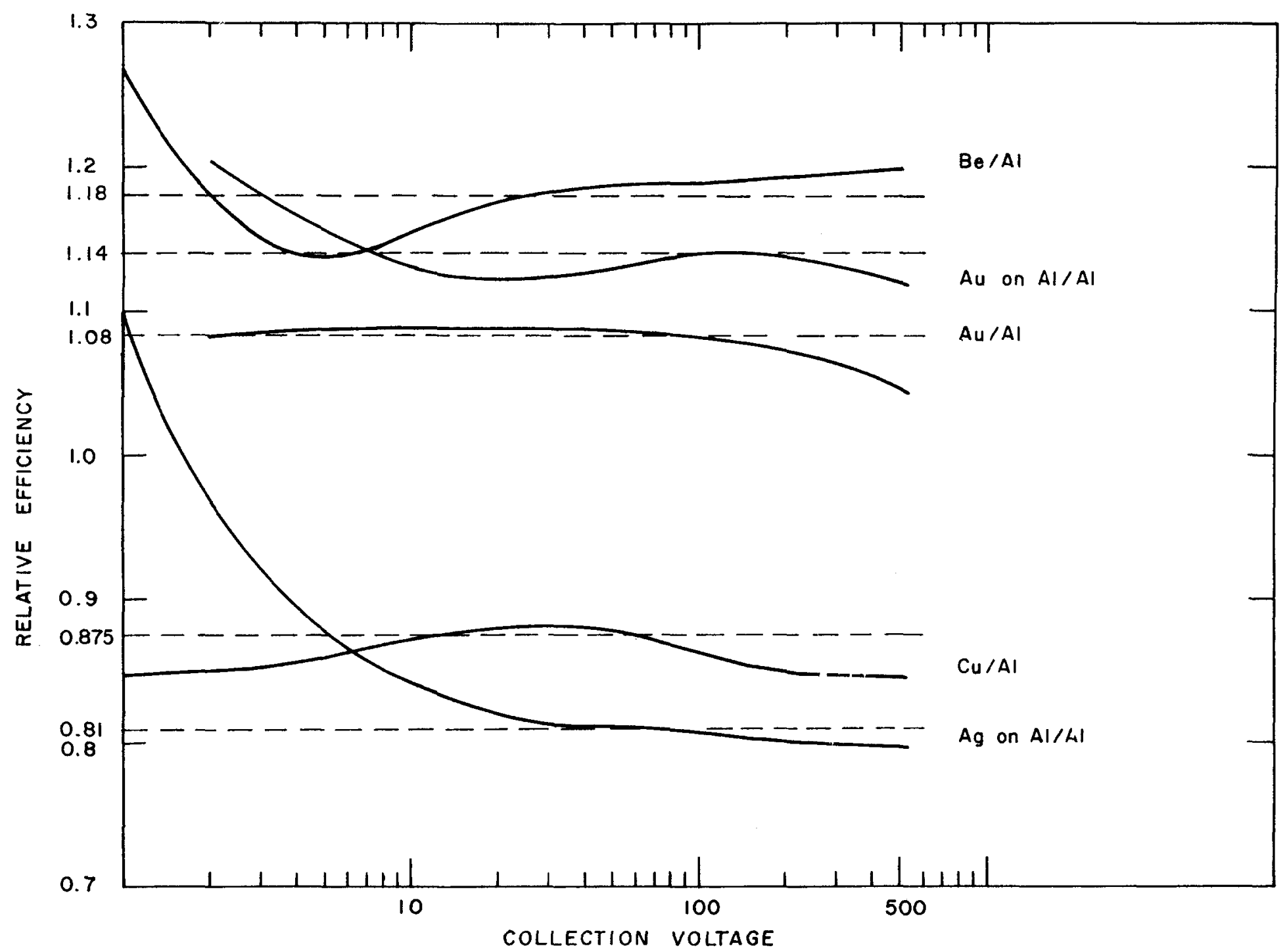

FIG. 6 - RELATIVE EFFICIENCY VS. COLLECTION VOLTAGE 


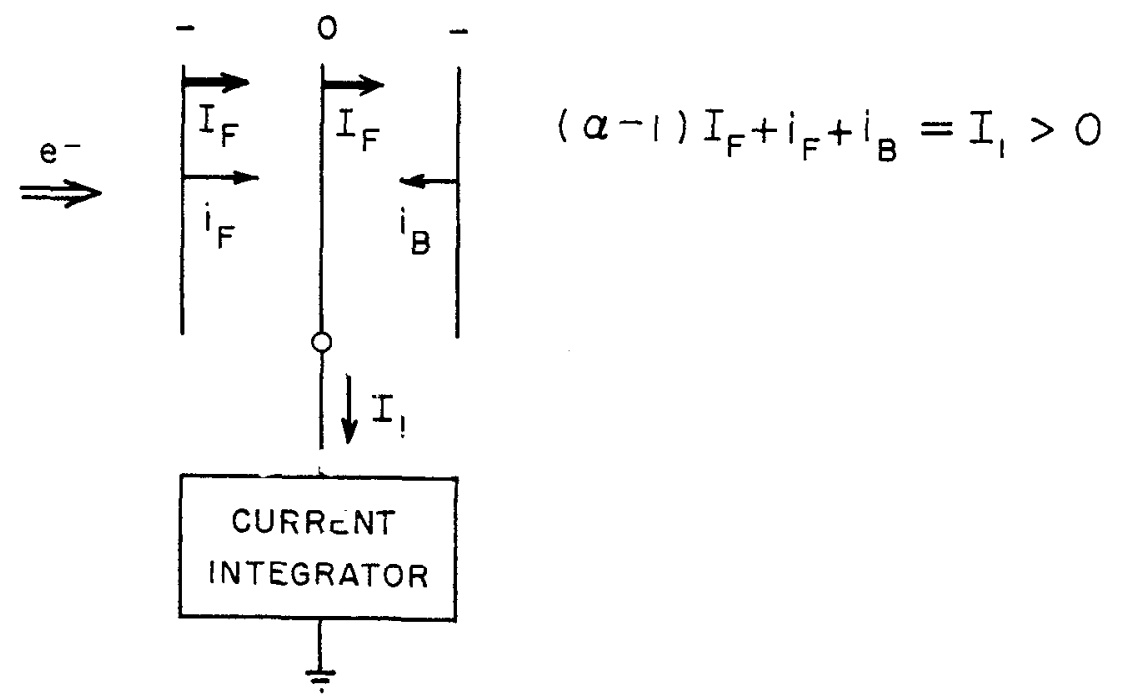

(a)

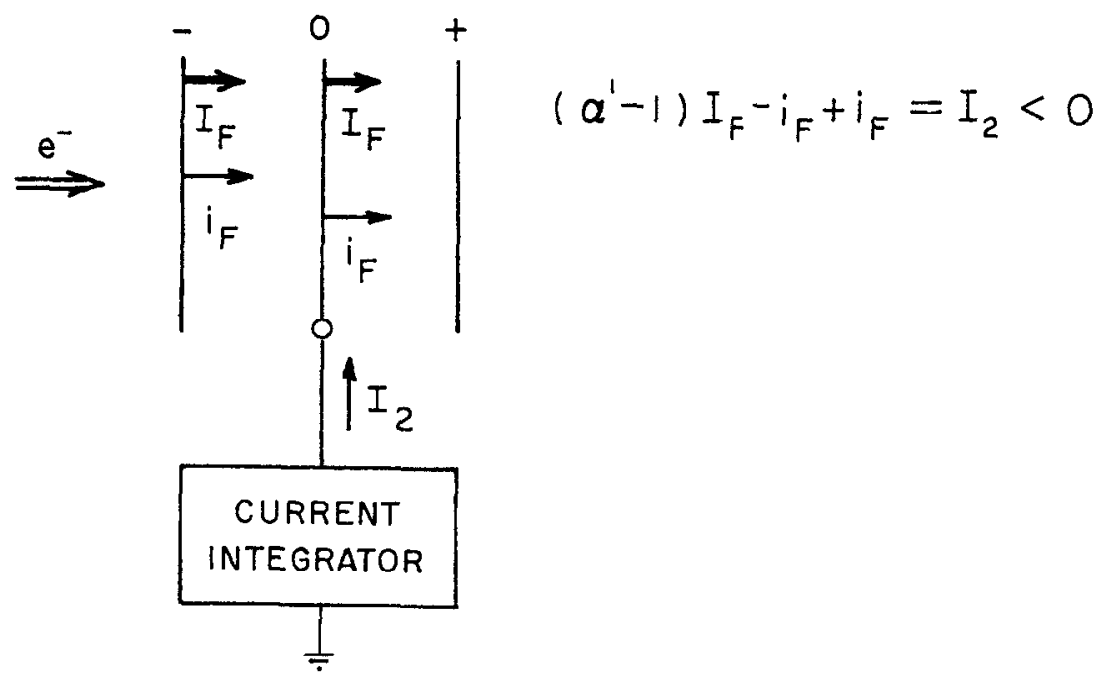

(b)

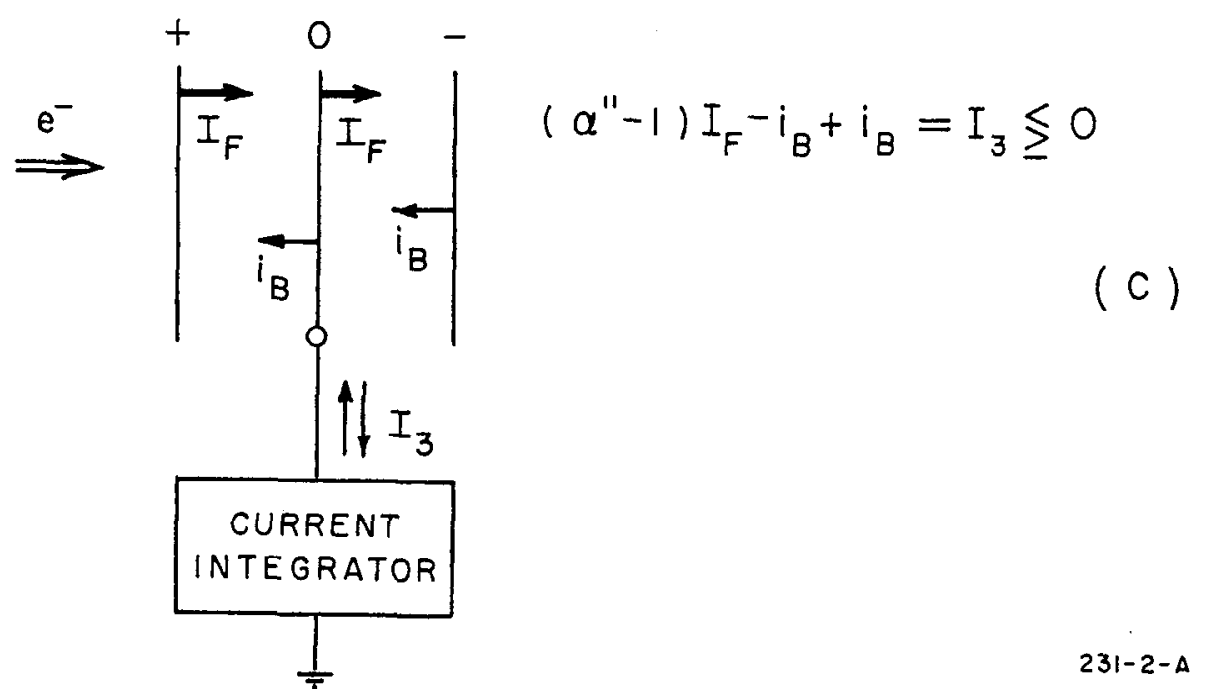

FIG.7 - ARRANGEMENTS FOR MEASURING THE HIGH ENERGY COMPONENTS IN THE SECONDARY ELECTRON EMISSION. 


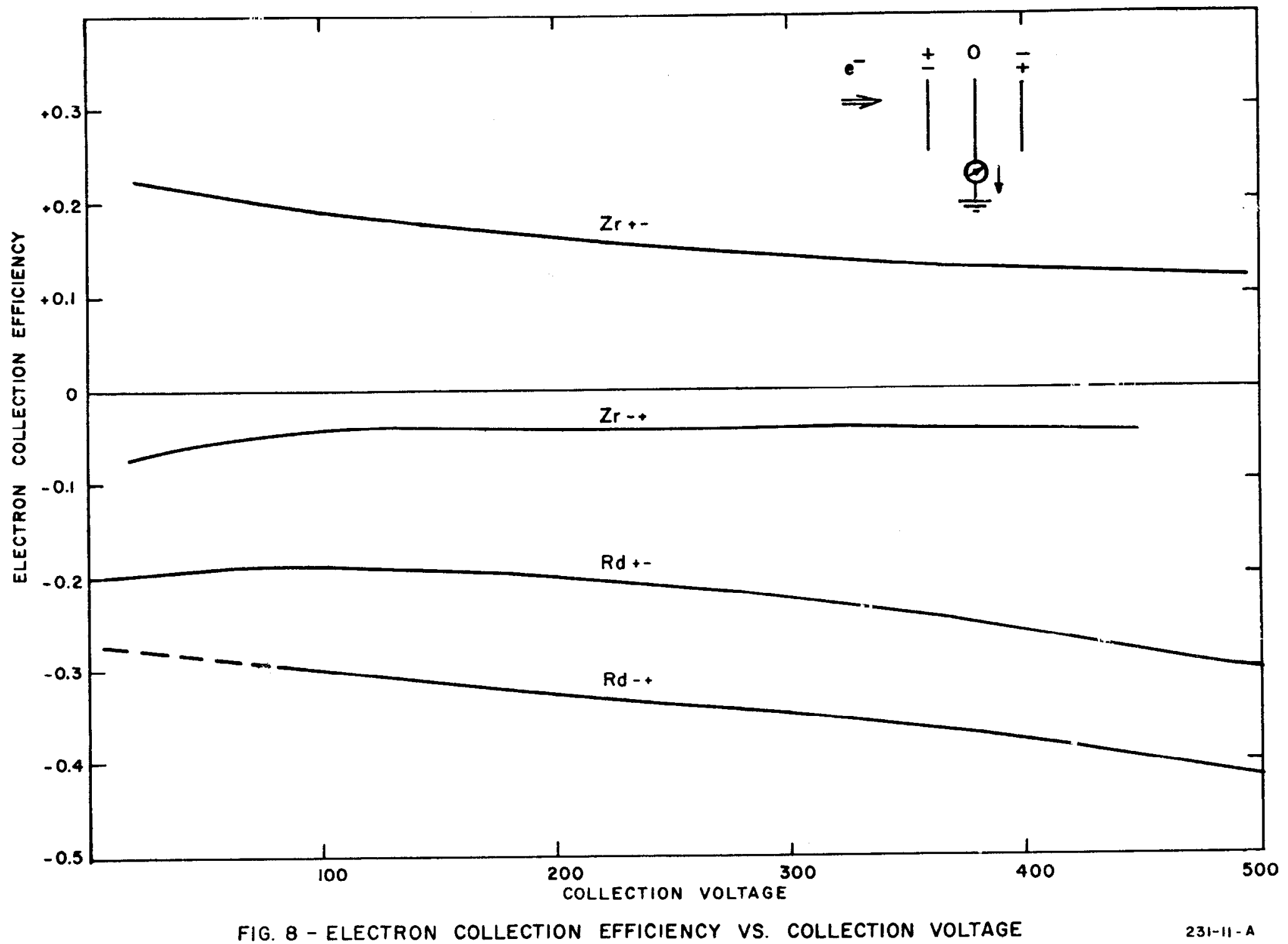

